SLAC-PUB-8160

June 1999

\title{
A STUDY OF CORRELATIONS BETWEEN IDENTIFIED CHARGED HADRONS IN HADRONIC $Z^{0}$ DECAYS*
}

\author{
The SLD Collaboration** \\ Stanford Linear Accelerator Center \\ Stanford University, Stanford, CA 94309
}

\begin{abstract}
We present a preliminary study of correlations in rapidity between pairs of identified charged pions, kaons and protons using the entire SLD data sample of 550,000 hadronic $Z^{0}$ decays. Short range charge correlations are observed between all combinations of these hadron species, indicating local conservation of quantum numbers and charge ordering in the jet fragmentation process. The rapidity range of this effect is found to be independent of particle momentum. A strong long-range $K^{+}-K^{-}$correlation is observed at high-momentum and weaker long-range $\pi^{+}-\pi^{-}, \pi^{+}-K^{-}$and $\mathrm{p}-K^{-}$and $\mathrm{p} \overline{\mathrm{p}}$ correlations are observed in light flavor events, providing new information on leading particle production in $u, d$ and $s$ jets. The longrange correlations observed in $c \bar{c}$ and $b \bar{b}$ events are markedly different and consistent with expectations based on known decay properties of the leading heavy hadrons. In addition, the SLC electron beam polarization is used to tag the quark hemisphere in each event, allowing the first study of rapidities signed such that positive rapidity is along the quark rather than antiquark direction. Distributions of ordered differences in signed rapidity between pairs of particles provide a direct probe of quantum number ordering along the quark-antiquark axis and other new insights into the fragmentation process.
\end{abstract}

Contributed to: the International Europhysics Conference on High Energy Physics, 1521 July 1999, Tampere, Finland; Ref: 1_187, and to the XIX ${ }^{\text {th }}$ International Symposium on Lepton Photon Interactions, August 9-14, 1999, Stanford, USA.

*Work supported by Department of Energy contract DE-AC03-76SF00515. 


\section{Introduction}

Correlations between particles produced in hadronic jets can be used to probe details of the jet fragmentation process. In $e^{+} e^{-}$annihilations into hadrons, the total charge, strangeness, baryon number, etc. of the final state particles in each event must be zero, and it is interesting to ask whether the conservation of such quantum numbers is local, or is longer-range in character. For example, in the case of strangeness, one can ask whether a strange particle and a corresponding antistrange particle tend to be produced "close" to each other within the event, whether strange and antistrange particles are associated with the initial $\bar{s}$ and $s$ quarks, respectively, in $s \bar{s}$ events (or with the initial $u$ and $\bar{u}$ in $u \bar{u}$ events, etc.), or whether strange and antistrange particles are distributed randomly throughout the event. Similar questions can be posed for other relevant quantum numbers. Previous studies [1] of differences in rapidity between associated identified hadrons have shown that the conservation of charge, strangeness and baryon number is predominantly local. Most fragmentation models implicitly implement this feature, and the form and range of such short-range correlations provide useful tests of these models. Short-range correlations can also arise from the decays of heavier hadrons, for example the decay $\rho^{0} \rightarrow \pi^{+} \pi^{-}$will produce opposite-charge pion pairs with a characteristic degree of locality.

Long-range correlations between particles of opposite charge and strangeness in opposite jets of an event have also been observed [2]. These can be understood in terms of leading particle production whereby the higher-rapidity tracks in each jet tend to carry the quantum numbers of the initial quark or antiquark. In the case of $e^{+} e^{-} \rightarrow s \bar{s}$ events, the $s$ and $\bar{s}$ quarks may hadronize, for example, into a high momentum $K^{-}$ and $K^{+}$, respectively, and there need be no other strange particles in the event. In $u \bar{u}$ and $d \bar{d}$ events, however, the locality of quantum number conservation implies that a high-momentum strange-antistrange pair must be produced in each jet, which will dilute any long-range correlation. Nevertheless, improved measurements of long-range correlations may provide better understanding of leading particle production.

The rapidity of a particle is typically defined with an arbitrary sign. If a sign could be given to each measured rapidity such that, for example, positive (negative) rapidity corresponds to the initial quark (antiquark) direction, then one might probe more deeply into both leading and nonleading particle production. One could measure, for 
example, the extent to which a leading particle has higher rapidity than its associated antiparticle, and the extent to which low-momentum particles in jets remember the initial quark/antiquark direction.

In this paper we present a study of correlations in rapidity between identified charged pions, kaons and protons based on about 550,000 hadronic $Z^{0}$ decays recorded by the SLD experiment at the SLAC Linear Collider. Clean samples of identified particles were obtained using the Cherenkov Ring Imaging Detector. The hadronic event

sample was divided into samples enriched in light-flavor $\left(e^{+} e^{-} \rightarrow u \bar{u}, d \bar{d}\right.$ or $\left.s \bar{s}\right), c \bar{c}$ and $b \bar{b}$ events in order to study the effects of the decays of the leading bottom and charmed hadrons. In section 4 we present a study of short-range correlations between all pair-combinations of these hadron species as a function of hadron momentum. We quantify the range of each correlation, and compare with the predictions of the JETSET fragmentation model. In section 5 we search for long-range correlations between these species. In section 6 we exploit the SLC electron beam polarization to identify the quark (vs. antiquark) hemisphere in each event and sign the rapidities such that particles in the quark-tagged hemisphere have positive rapidity. The signed rapidity distributions themselves provide new information on leading particle production, and ordered rapidity differences between particle pairs allow new and unique probes of the fragmentation process.

\section{Apparatus and Hadronic Event Selection}

A general description of the SLD can be found elsewhere [3]. The trigger and initial selection criteria for hadronic $Z^{0}$ decays are described in Ref. [4]. This analysis used charged tracks measured in the Central Drift Chamber (CDC) [5] and Vertex Detector (VXD) [6], and identified using the Cherenkov Ring Imaging Detector (CRID) [7]. Momentum measurement is provided by a uniform axial magnetic field of $0.6 \mathrm{~T}$. The $\mathrm{CDC}$ and VXD give a momentum resolution of $\sigma_{p_{\perp}} / p_{\perp}=0.01 \oplus 0.0026 p_{\perp}$, where $p_{\perp}$ is the track momentum transverse to the beam axis in $\mathrm{GeV} / c$. One quarter of the data were taken with the original vertex detector (VXD2), and the rest with the upgraded detector (VXD3). In the plane normal to the beamline the centroid of the micronsized SLC IP was reconstructed from tracks in sets of approximately thirty sequential 
hadronic $Z^{0}$ decays to a precision of $\sigma_{I P} \simeq 7 \mu \mathrm{m}$ for the VXD2 data and $\simeq 3 \mu \mathrm{m}$ for the VXD3 data. Including the uncertainty on the IP position, the resolution on the charged track impact parameter $(\delta)$ projected in the plane perpendicular to the beamline is $\sigma_{\delta}=11 \oplus 70 /\left(p \sin ^{3 / 2} \theta\right) \mu \mathrm{m}$ for VXD2 and $\sigma_{\delta}=8 \oplus 29 /\left(p \sin ^{3 / 2} \theta\right) \mu \mathrm{m}$ for VXD3, where $\theta$ is the track polar angle with respect to the beamline. The CRID comprises two radiator systems that allow the identification of charged pions with high efficiency and purity in the momentum range $0.3-35 \mathrm{GeV} / \mathrm{c}$, charged kaons in the ranges $0.75-6 \mathrm{GeV} / \mathrm{c}$ and $9-35 \mathrm{GeV} / \mathrm{c}$, and protons in the ranges $0.75-6 \mathrm{GeV} / \mathrm{c}$ and 10-46 GeV/c [8]. The event thrust axis [9] was calculated using energy clusters measured in the Liquid Argon Calorimeter [10].

A set of cuts was applied to the data to select well-measured tracks and events well contained within the detector acceptance. Charged tracks were required to have a distance of closest approach transverse to the beam axis within $5 \mathrm{~cm}$, and within $10 \mathrm{~cm}$ along the axis from the measured IP, as well as $|\cos \theta|<0.80$, and $p_{\perp}>0.15 \mathrm{GeV} / \mathrm{c}$. Events were required to have a minimum of seven such tracks, a thrust axis polar angle w.r.t. the beamline, $\theta_{T}$, within $\left|\cos \theta_{T}\right|<0.71$, and a charged visible energy $E_{v i s}$ of at least $20 \mathrm{GeV}$, which was calculated from the selected tracks assigned the charged pion mass. The efficiency for selecting a well-contained $Z^{0} \rightarrow q \bar{q}(g)$ event was estimated to be above $96 \%$ independent of quark flavor. The VXD, CDC and CRID were required to be operational, resulting in a selected sample of roughly 285,000 events, with an estimated non-hadronic background contribution of $0.10 \pm 0.05 \%$ dominated by $Z^{0} \rightarrow \tau^{+} \tau^{-}$events.

Samples of events enriched in light and $b$ primary flavors were selected based on charged track impact parameters $\delta$ with respect to the IP in the plane transverse to the beam [11]. For each event we define $n_{\text {sig }}$ as the number of tracks with impact parameter greater than three times its estimated error, $\delta>3 \sigma_{\delta}$. Events with $n_{\text {sig }}=0$ were assigned to the light-flavor sample and those with $n_{\text {sig }} \geq 4$ were assigned to the $b$-flavor sample; the remaining events were classified as a $c$-flavor sample. The light-, $c$ - and b-tagged samples comprised 166,000, 82,000 and 37,000 events, respectively; selection efficiencies and sample purities were estimated from our Monte Carlo simulation and are listed in table 1 .

Separate samples of hemispheres enriched in quark and antiquark jets were selected 


\begin{tabular}{|l||c|c|c||c|c|c|}
\hline \multicolumn{1}{|c||}{} & \multicolumn{3}{c||}{ Efficiency for $Z^{0} \rightarrow$} & \multicolumn{3}{c|}{ Purity of $Z^{0} \rightarrow$} \\
& $u \bar{u}, d \bar{d}, s \bar{s}$ & $c \bar{c}$ & $b \bar{b}$ & $u \bar{u}, d \bar{d}, s \bar{s}$ & $c \bar{c}$ & $b \bar{b}$ \\
\hline light-tag & 0.846 & 0.338 & 0.034 & 0.881 & 0.106 & 0.013 \\
$c$-tag & 0.153 & 0.617 & 0.401 & 0.320 & 0.388 & 0.292 \\
$b$-tag & 0.001 & 0.045 & 0.565 & 0.005 & 0.064 & 0.931 \\
\hline
\end{tabular}

Table 1: Tagging efficiencies for simulated events in the three flavor categories to be tagged as light, $c$ or $b$. The three rightmost columns indicate the composition of each simulated tagged sample assuming SM relative flavor production.

by exploiting the large electroweak forward-backward production asymmetry wrt the beam direction. The event thrust axis was used to approximate the initial $q \bar{q}$ axis and was signed such that its $z$-component was positive, $\hat{t}_{z}>0$. Events in the central region of the detector, where the production asymmetry is small, were removed by the requirement $\left|\hat{t}_{z}\right|>0.2$, leaving 235,000 events. The quark-tagged hemisphere in events with left-(right-)handed electron beam was defined to comprise the set of tracks with positive (negative) momentum projection along the signed thrust axis. The remaining tracks in each event were defined to be in the antiquark-tagged hemisphere. The sign and magnitude of the electron beam polarization were measured for every event. For the selected event sample, the average magnitude of the polarization was 0.73 . Using this value and assuming Standard Model couplings at tree-level, the purity of the quark-tagged sample is 0.73 .

For the purpose of estimating the efficiency and purity of the event flavor tagging and the particle identification, we made use of a detailed Monte Carlo (MC) simulation of the detector. The JETSET 7.4 [12 event generator was used, with parameter values tuned to hadronic $e^{+} e^{-}$annihilation data [13], combined with a simulation of $B$ hadron decays tuned [14] to $\Upsilon(4 S)$ data and a simulation of the SLD based on GEANT 3.21 15. Inclusive distributions of single-particle and event-topology observables in hadronic events were found to be well described by the simulation [4]. 


\section{Identified Particle Selection}

The identification of charged tracks as pions, kaons or protons using the CRID is described in detail in [8]. For this analysis we used a relatively loose identification algorithm, since the presence of misidentified hadrons at the $10 \%$ level has little effect on the measured correlations. Tracks with poor CRID information or that were likely to have scattered or interacted before exiting the CRID were removed by requiring each track to have at least $40 \mathrm{CDC}$ hits, at least one of which was at a radius of at least $92 \mathrm{~cm}$, to extrapolate through an active region of the appropriate CRID radiator and through a live CRID TPC, and, in the case of the gas radiator, to have fewer than four saturated hits within the volume in which the gas ring is expected. Approximately $85 \%$ of the tracks within the CRID acceptance satisfied these cuts.

Tracks identified in the calorimeters as electrons or muons [1]6] were rejected, and for the remaining tracks log-likelihoods [8, 17] were calculated for each of the three charged hadron hypotheses $i=\pi, K$ and $\mathrm{p}$, and for each of the liquid and gas radiators. A track was tagged as a hadron of species $i$ by the liquid (gas) system if the log-likelihood for hypothesis $i$ exceeded both of the other log-likelihoods by at least 5 (3) units. In addition, for those tracks with good information from both the liquid and gas systems, the liquid and gas log-likelihoods were added together, and a track was tagged by the combined system if the log-likelihood for hypothesis $i$ exceeded both of the others by at least 3 units. A track was identified as an $i$-hadron if it was tagged as type $i$ by any of the liquid, gas or combined systems, and it was not tagged as any other type by any other system. The efficiencies for identifying accepted tracks are similar to those given in [8]: for charged pions there is roughly constant efficiency of about $80 \%$ in the momentum range $0.5-25 \mathrm{GeV} / \mathrm{c}$; charged kaons and protons have similar efficiency except for a dip in the range $5-10 \mathrm{GeV} / \mathrm{c}$. The simulation was found to provide a good description of the momentum distributions of the identified hadrons.

For each identified track the rapidity $y=0.5 \ln \left(\left(E+p_{\|}\right) /\left(E-p_{\|}\right)\right)$was calculated using the measured momentum and its projection $p_{\|}$along the thrust axis, and the appropriate hadron mass. The distributions of rapidity are shown in fig. 1 for each identified hadron species, along with prediction of the simulation. Note that the overall sign of the thrust axis vector, and therefore the sign of the rapidity, is arbitrary. These distributions are not flat in the central region, but show structure due to the 


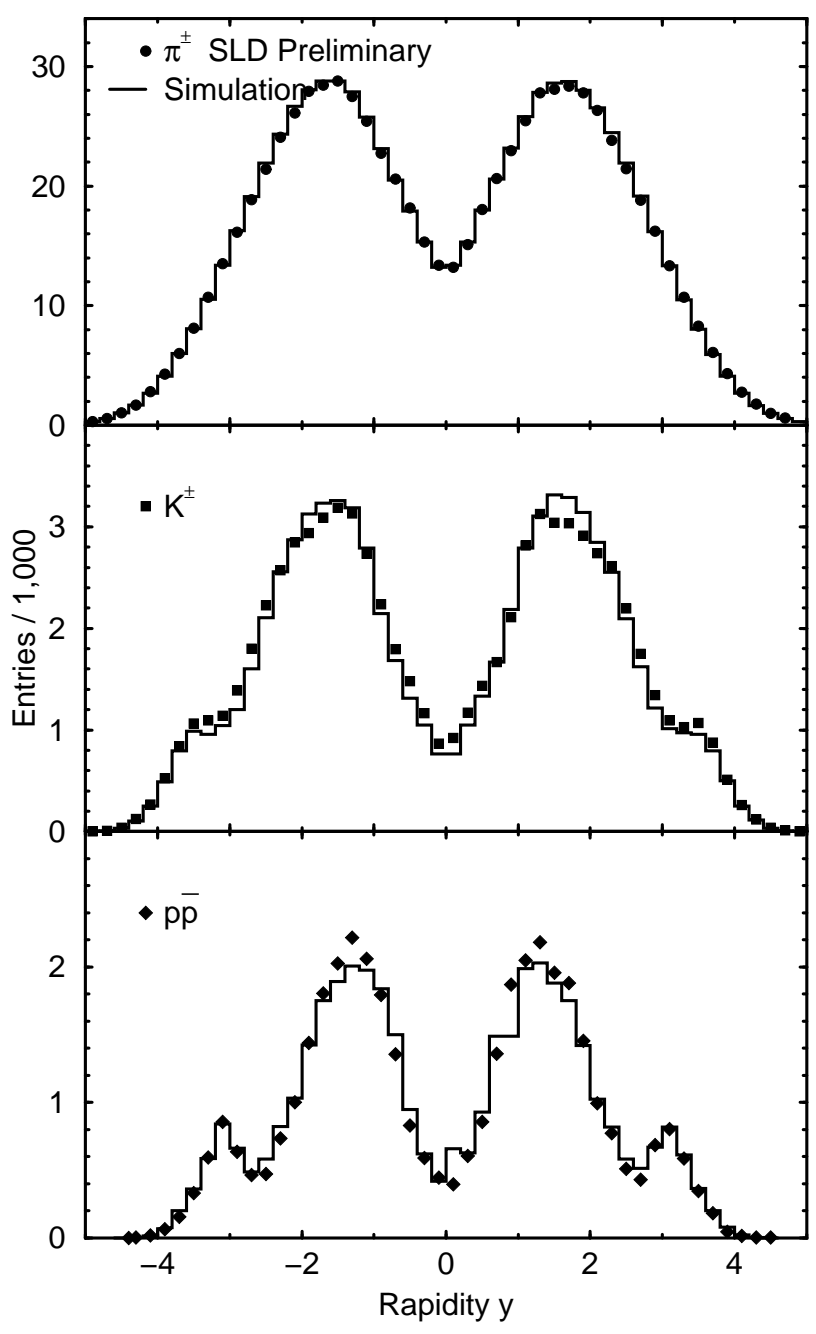

Figure 1: Rapidity distributions for the identified pions, kaons and protons. Also shown are the prediction of the Monte Carlo simulation.

momentum dependence of the CRID identification efficiency (see [8]). The simulation provides a good qualitative description of the rapidity distributions; the dependence of the identification efficiency on momentum has only modest effects on the correlation studies, as discussed below.

The absolute value of the difference between the rapidities of each pair of identified particles was calculated, and the distribution of this quantity is shown in fig. 22 for each of the six possible pairs of hadron species. In each case the distribution for those pairs with opposite charge is shown as the solid histogram and that for pairs with the same charge is shown as the dashed histogram. 


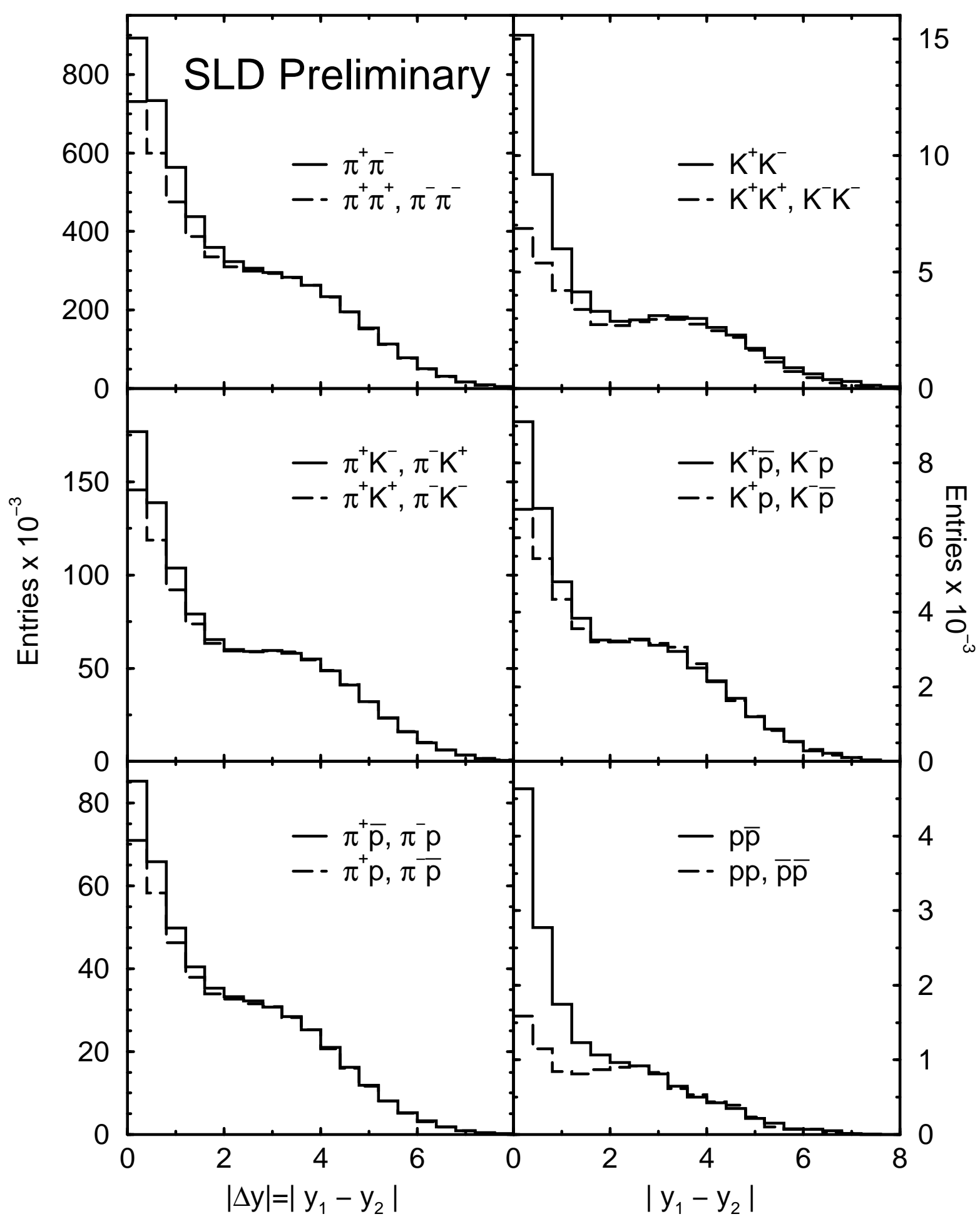

Figure 2: Rapidity difference distributions for opposite-charge (histograms) and samecharge (dashed histograms) pairs of identified pions, kaons and protons. 


\section{Short-Range Correlations}

For every type of hadron pair in fig. 2 there is an excess of opposite-charge pairs over same-charge pairs at small values of the absolute rapidity difference $|\Delta y|=\left|y_{1}-y_{2}\right|$. We expect more opposite-charge than same-charge track pairs, as well as more $K \bar{K}$ than $K K / \bar{K} \bar{K}$ pairs and more $\bar{p} \overline{\mathrm{p}}$ than $\mathrm{pp} / \overline{\mathrm{p}} \overline{\mathrm{p}}$ pairs, due to conservation of electric charge, strangeness and baryon number, respectively. In the case of $K K(\mathrm{pp})$ pairs the size of the excess is sensitive to the relative fraction of strange-antistrange (baryonantibaryon) pairs that are both charged rather than neutral; if e.g. p- $\bar{n}$ were the only type of baryon-antibaryon pair produced, there would be no such excess. The fact that the excess of opposite-charge $K K$ and pp pairs peaks at low values of $|\Delta y|$ indicates that conservation of strangeness and baryon number, respectively, is local, as has been observed previously [1].

In order to study these short-range correlations in more detail, we assumed that the tracks in each same-charge pair are unassociated, and subtracted their $|\Delta y|$ distributions from those of the respective opposite-charge pairs. These differences are shown for the low $|\Delta y|$ region in fig. 3, and are seen to differ significantly from each other in form. Monte Carlo studies indicate that these differences are not due to acceptance, momentum dependence of the particle identification efficiency or to background from misidentified particles. Also shown in fig. 司 are the predictions of the simulation for this difference. In all cases, the simulation gives a reasonable description of the form of the difference, although there are small differences in the details of the form for $\pi \pi$, $\pi \mathrm{p}, K K$ and $\pi \mathrm{p}$ pairs. The predictions for the amplitudes have $\sim 5 \%$ normalization uncertainties (not shown) due to uncertainties in the particle identification efficiencies. The predicted amplitudes are thus consistent with the data, except that for the $K \mathrm{p}$ correlation, which is low by about $40 \%$.

Pairs of tracks from the decays of resonances (e.g. $\rho^{0} \rightarrow \pi^{+} \pi^{-}$) contribute to varying degrees for all pair combinations; each decay mode gives a $|\Delta y|$ difference distribution with a characteristic form, and collectively they have a substantial influence on the overall form of the distributions. Since the resonance production rates are adjustable in the simulation, we suspect that it is able to provide an adequate description of the data.

Pairs in which one or both particles are misidentified are an important source of 
background, and lead to a convergence in the forms of the distributions for different pair combinations. The simulated differences for those pairs in which at least one track is misidentified are also shown in fig. 3, and are seen to contribute a sizeable fraction of the observed differences in the cases of unlike particles $(\pi K, \pi \mathrm{p}$ and $K \mathrm{p})$. There are normalization uncertainties on these predictions of $\sim 20 \%$ due to uncertainties in the misidentification rates. Excellent particle identification is required to demonstrate, as seen in the figure, that there are excesses at short range for true pairs of these three combinations, indicating local conservation of electric charge between these different particle species and suggesting that there is charge ordering among hadrons of all species in the fragmentation process. The prediction of the simulation for the observed $K \mathrm{p}$ correlation is dominated by misidentification; the prediction for the true correlation is therefore low by a factor of $\sim 3$.

We have studied these short-range correlations in 6 bins of the momentum of the heavier (higher momentum) particle in the case of $\pi K, \pi \mathrm{p}$ and $K \mathrm{p}(\pi \pi, K K$ and pp) pairs. We find the quality of the simulated description of the data to be independent of the momentum bin. In order to quantify the range of each correlation, we fitted an ad hoc function, the sum of two Gaussians, to each difference in each bin over the range $0<|\Delta y|<3$ units. The centers of both Gaussians were fixed to zero, and the amplitude (width) of the wider Gaussian was fixed to 0.4 (2.2) times that of the narrower Gaussian, leaving the amplitude and width of the narrower Gaussian as free parameters. We used the width as a measure of the range of the correlation. This function provided a reasonable qualitative description of both the data and simulation for all pair combinations in all momentum bins.

The fitted widths are shown in fig. 1 as a function of momentum for each pair combination. If the fragmentation process is scale invariant, then we expect the range to be independent of momentum, except for biases introduced by the bin edges and the particle identification. In the case of $\pi \pi$, the range is constant within ten percent except for the highest momentum bin. Significant momentum dependence is observed for $\pi p$, $K K, K \mathrm{p}$ and pp pairs, however this dependence is reproduced by the simulation, with the possible exception of differences at low momentum for $\pi \mathrm{p}$ and $K \mathrm{p}$ pairs, and at high momentum for $K K$ pairs. In the simulation, there is a $\sim 10 \%$ momentum dependence due to binning and particle decays. The large slopes and point-to-point structure 


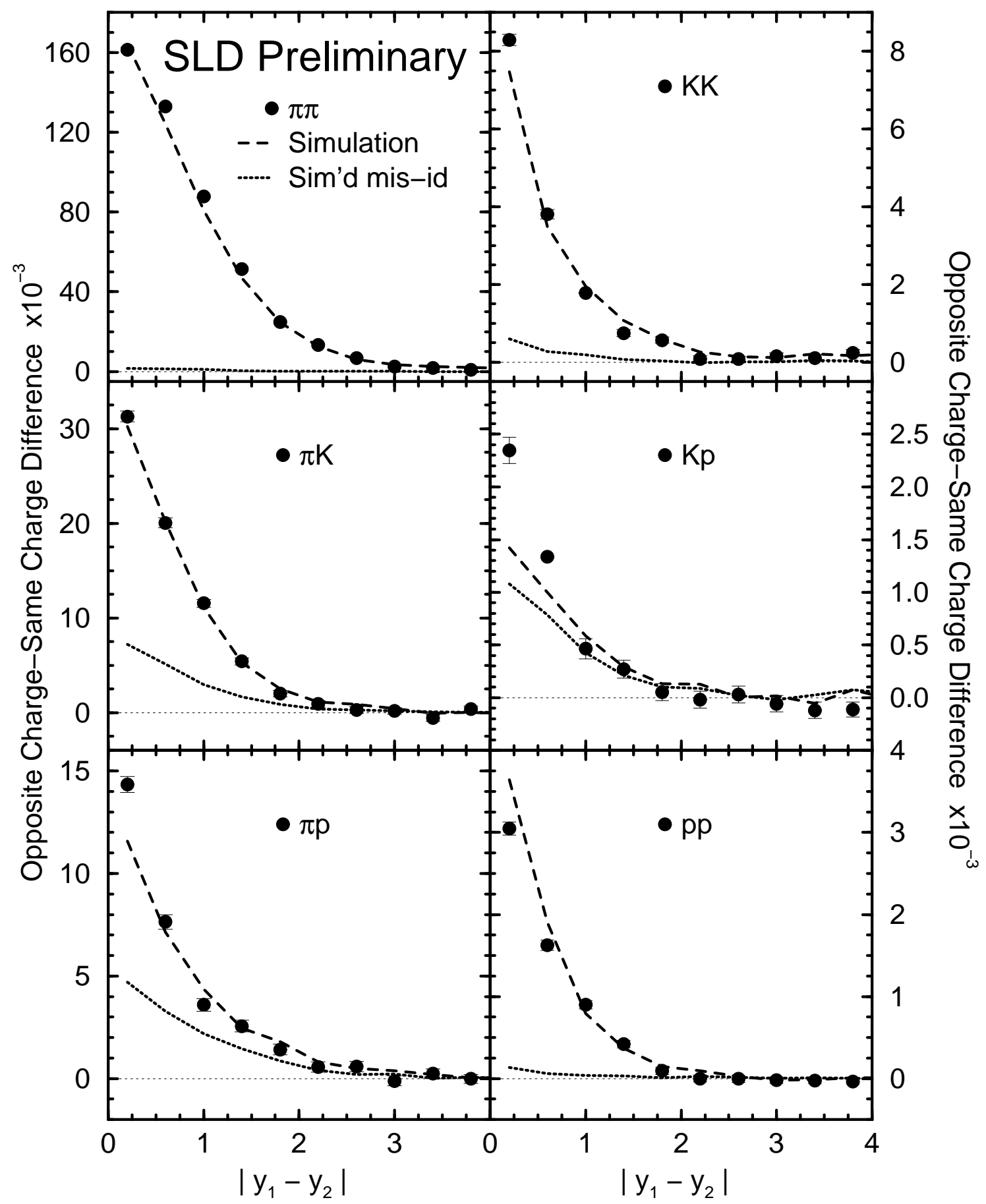

Figure 3: Differences between the $|\Delta y|$ distributions for opposite-charge and samecharge pairs in the small $|\Delta y|$ region. The errors are statistical only. Also shown are the predictions of the Monte Carlo simulation for all detected pairs (dashed lines) and those pairs in which one or both particles were misidentified (dotted lines). There are normalization uncertainties of $\sim 5 \%$ on the total predictions and $\sim 20 \%$ on the component due to misidentified particles. 


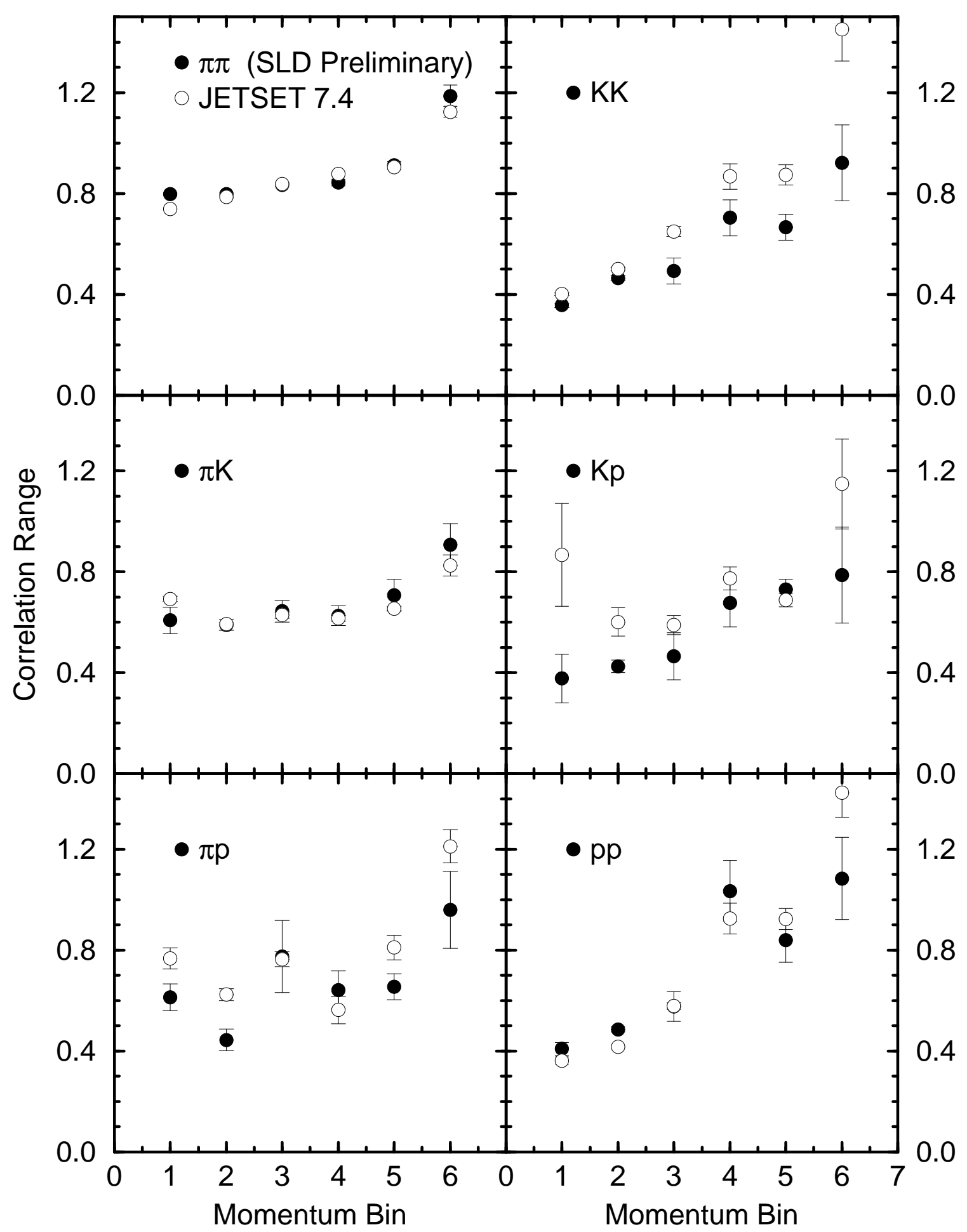

Figure 4: Range (see text) of the observed short-range correlations in the data (dots) and simulation (open circles), in six bins of momentum for each pair combination. 
are due to the momentum dependence of the particle identification efficiencies. Thus the hypothesis of momentum-independence of the range of the short-range rapidity correlation for a given pair combination is consistent with the data, within the context of the JETSET model.

The qualitative results presented above were found to be independent of the primary flavor of the event, as were the quality of the predictions of the simulation and the feature of scale independence. This is expected, as the only difference at short range should be due to the decay properties of the leading $B$ and $D$ hadrons; we do observe some small differences between the flavor-tagged samples in the measured ranges of the correlations, which are reproduced by the simulation.

\section{Long-Range Correlations}

We next searched for long-range correlations between all pair combinations. In fig. 2, a difference between opposite-charge and same-charge pairs at high $|\Delta y|$ is visible only in the case of $K K$, and even here the background from uncorrelated pairs is dominant. Since we expect long-range correlations from leading particle production to be relatively more important at high momentum, we have studied these differences for pairs in which both tracks have momentum $p>9 \mathrm{GeV} / \mathrm{c}$. The corresponding $|\Delta y|$ distributions for each of the six pair combinations are shown in fig. 5. For these high-momentum pairs, there is a separation between pairs in the same jet $(|\Delta y|<2.5)$ and those in opposite jets $(|\Delta y|>4)$. At short range there is a large excess of opposite-charge pairs over same-charge pairs for all pair combinations, confirming that locality holds even at the highest momenta. At long range, there are clear correlations for $\pi \pi$ and $K K$ pairs, as well as significant correlations for $\pi \mathrm{p}, K \mathrm{p}$ and pp pairs.

These long-range correlations are found to be strongly flavor-dependent. Figure 6 shows the differences between the opposite-charge and same-charge pairs in the high $|\Delta y|$ region for each of the flavor-tagged samples. The $b \bar{b}$ events are seen to contribute very little to the difference for any pair combination, primarily because there are relatively few tracks with such high momentum in these events. Light flavor and $c \bar{c}$ events contribute roughly equally to the observed correlations for $\pi \pi, \pi \mathrm{p}$ and pp pairs; lightflavor events dominate the $K \mathrm{p}$ correlation. In the case of $\pi \mathrm{K}$ pairs, there is a strong 


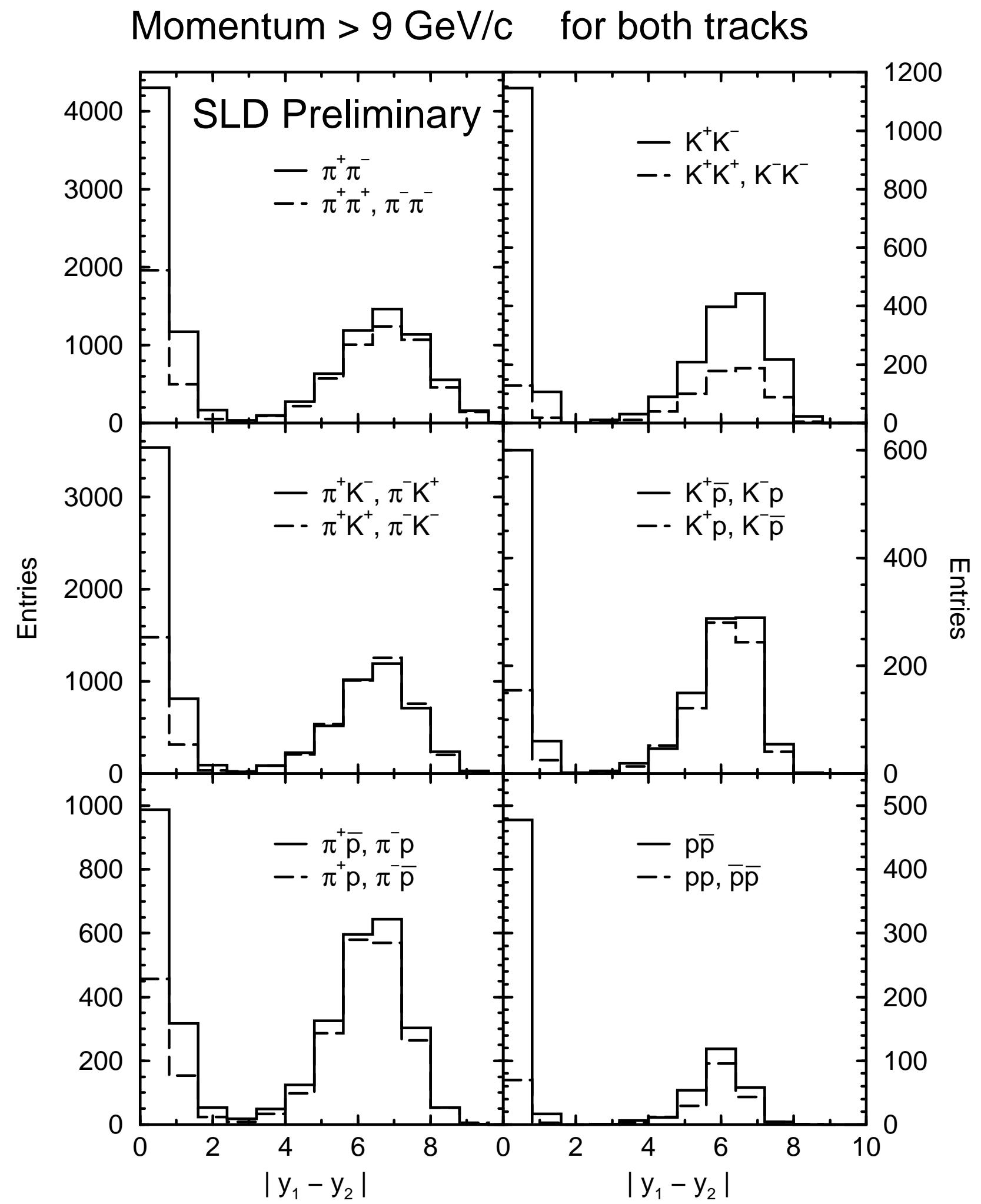

Figure 5: Observed $|\Delta y|$ distributions for opposite-charge (histograms) and samecharge (dashed histograms) pairs in which both tracks have $p>9 \mathrm{GeV} / \mathrm{c}$. 


\begin{tabular}{|c||r|r|r||r|r|r|}
\hline \multicolumn{1}{|c||}{$\begin{array}{c}\text { Pair } \\
\text { Type }\end{array}$} & \multicolumn{3}{c||}{ Data } & \multicolumn{3}{c|}{ Simulation } \\
\hline$\pi \pi$-tag & $c$-tag & $b$-tag & $u d s$-tag & $c$-tag & $b$-tag \\
\hline$\pi$ & $398 \pm 85$ & $265 \pm 46$ & $50 \pm 27$ & $488 \pm 42$ & $241 \pm 23$ & $41 \pm 10$ \\
$\pi \mathrm{p}$ & $171 \pm 73$ & $-245 \pm 45$ & $15 \pm 25$ & $43 \pm 34$ & $-169 \pm 20$ & $20 \pm 9$ \\
$K K$ & $103 \pm 53$ & $93 \pm 28$ & $-5 \pm 15$ & $145 \pm 25$ & $44 \pm 13$ & $8 \pm 5$ \\
$K \mathrm{p}$ & $552 \pm 33$ & $192 \pm 20$ & $37 \pm 12$ & $449 \pm 16$ & $190 \pm 10$ & $28 \pm 4$ \\
$\mathrm{pp}$ & $39 \pm 1733$ & $1 \pm 20$ & $-11 \pm 10$ & $76 \pm 15$ & $1 \pm 9$ & $-6 \pm 3$ \\
\hline
\end{tabular}

Table 2: Observed differences between the numbers of opposite-charge and samecharge pairs in which both tracks had $p>9 \mathrm{GeV} / \mathrm{c}$ and $|\Delta y|>4$, in events in each of the three flavor-tagged samples. Also shown are the predictions of the Monte Carlo simulation.

anticorrelation in $c \bar{c}$ events along with a correlation in light-flavor events, both of which were invisible in the flavor-inclusive sample.

The integrated differences for $|\Delta y|>4$ are given for each flavor-tagged sample in table 2. The predictions of the simulation are also given and are generally consistent with the data. The simulation also describes the rapidity dependence in fig. 5 within the experimental uncertainties.

\section{6. $\quad$ Signed Rapidities and Correlations}

We next tagged the quark (vs. antiquark) direction in each hadronic event using the electron beam polarization for that event, exploiting the large forward-backward quark production asymmetry in $Z^{0}$ decays. If the beam was left-(right-)handed then the thrust axis was signed such that $\cos \theta_{T}$ was positive (negative). Events with $\left|\cos \theta_{T}\right|<$ 0.15 were removed, as the production asymmetry is small in this region. The probability to tag the quark direction correctly in these events was $73 \%$.

The rapidity of a particle with respect to the signed thrust axis is naturally signed such that positive rapidity corresponds to the hemisphere in the tagged direction of the initial quark, and negative rapidity corresponds to the tagged antiquark hemisphere. 


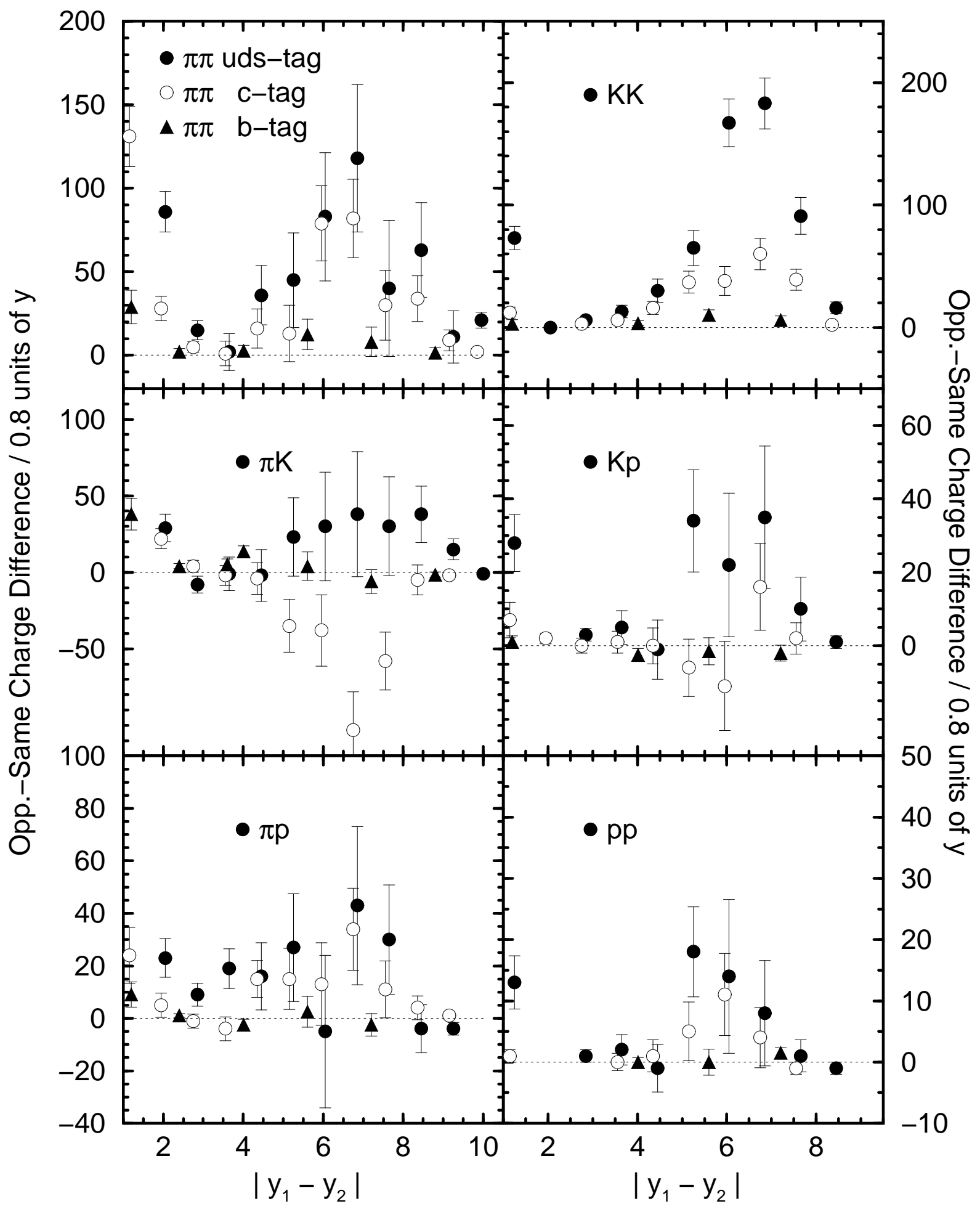

Figure 6: Observed differences between the $|\Delta y|$ distributions for opposite-charge and same-charge pairs in which both tracks have $p>9 \mathrm{GeV} / \mathrm{c}$, for the light-(dots), $c$ - (open circles) and $b$ - (triangles) tagged samples. 


\section{Charged Kaons}

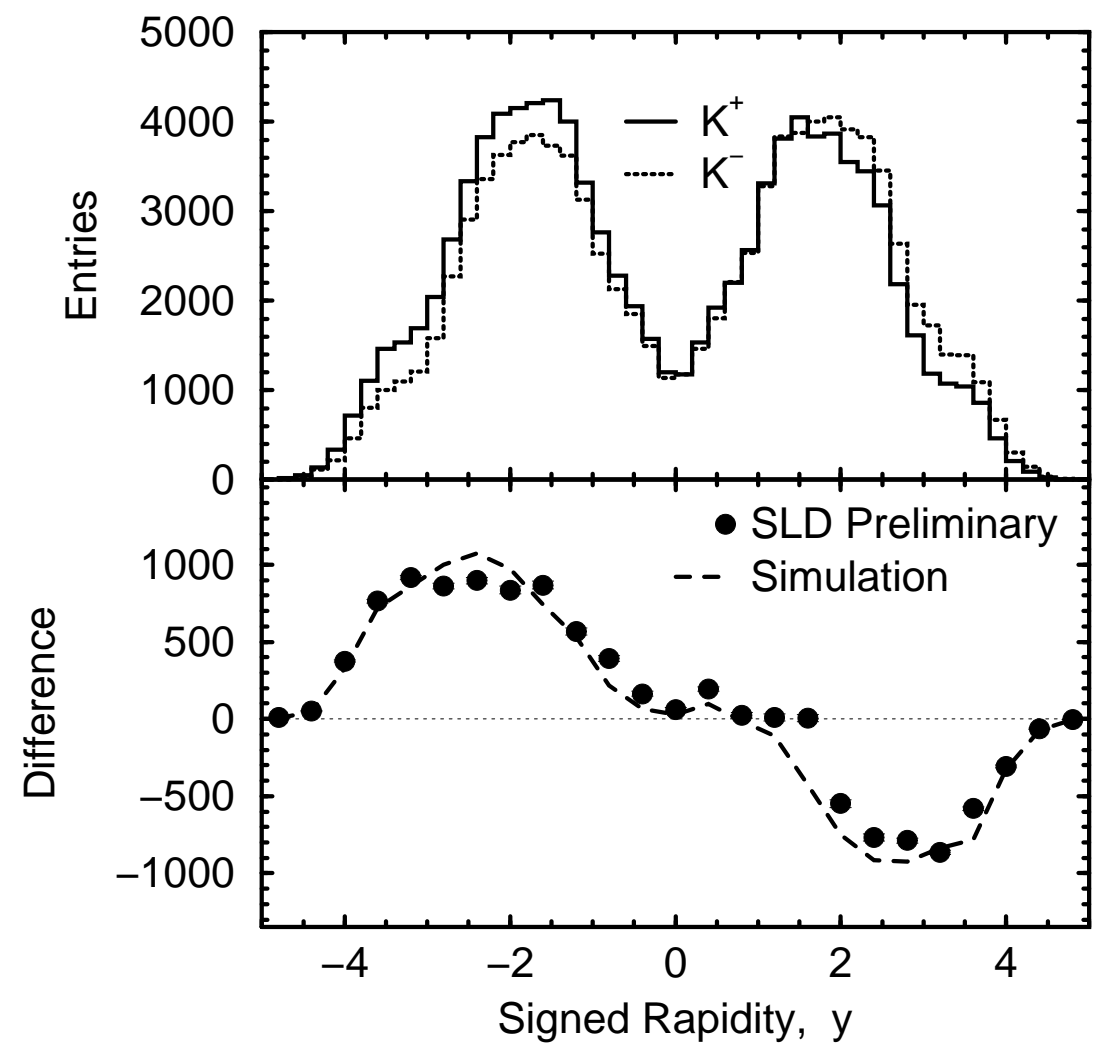

Figure 7: Distributions (top) of the rapidity with respect to the signed thrust axis for positively (histogram) and negatively (dashed histograms) charged kaons. The difference (bottom) between these two distributions compared with the prediction of the Monte Carlo simulation.

The signed rapidity distributions for identified $K^{+}$and $K^{-}$are shown in fig. 7. There is a clear difference between the two, with more $K^{-}$than $K^{+}$in the quark hemisphere, as expected due to leading $K^{-}$produced in $s$ quark jets [8, 18]. The difference between the two distributions is also shown in the figure and is compared with the prediction of the simulation, which is consistent with the data.

For pairs of identified particles, one can define an ordered rapidity difference. For particle-antiparticle pairs, we define $\Delta y^{+-}=y_{+}-y_{-}$as the difference between the signed rapidities of the positively charged particle and the negatively charged particle. In fig. 8 we show the distribution of $\Delta y^{+-}$for $\pi^{+} \pi^{-}, K^{+} K^{-}$and $\mathrm{p} \overline{\mathrm{p}}$ pairs. Asymmetries in these distributions are indications of ordering along the event axis, and the differences between the positive and negative sides of these distributions are also shown. The negative difference at high $\left|\Delta y^{+-}\right|$for the $K^{+} K^{-}$pairs can be attributed to the fact that leading kaons are produced predominantly in $s \bar{s}$ events. Similar but smaller effects for expected for $\pi^{+} \pi^{-}$and $\mathrm{p} \overline{\mathrm{p}}$ pairs, and the positive difference in the latter at $|\Delta y| \approx 4$ may be attributed to this effect. For $\pi^{+} \pi^{-}$pairs we observe a large positive difference at 
high $\left|\Delta y^{+-}\right|$rather than the expected small negative difference, which is due entirely to $c \bar{c}$ events (see below). The predictions of the simulation are also shown and are consistent with the data at high $\left|\Delta y^{+-}\right|$.

The positive difference in the lowest $\left|\Delta y^{+-}\right|$bins for the $\bar{p} \bar{p}$ pairs indicates that the baryon in an associated baryon-antibaryon pair follows the quark direction more closely than the antibaryon. This could be due to leading baryon production and/or to baryon number ordering along the entire fragmentation chain. We find a significant effect in all six of our momentum bins, and that the bulk of the observed difference occurs at low momentum (see fig. 9). We therefore conclude that both of these effects contribute; this is the first direct observation of baryon number ordering along the entire chain. Figure 9 also shows that the effect at long range noted above is confined to highmomentum pairs, as expected if due to leading baryon production. The prediction of the simulation is low by a factor of two at low $\left|\Delta y^{+-}\right|$in both fig. 8 and in the low $p_{\max }$ side of fig. 9; it is consistent with the high $p_{\max }$ data.

In fig. 10 we show the difference for $K^{+} K^{-}$pairs in two bins of $p_{\max }$ for the lightand $c$-tagged samples separately. For large $p_{\max }$ one can see differences in amplitude and in $|\Delta y|$ between the contributions from these two flavor-tagged samples. At low $p_{\max }$ there is a positive difference of three standard deviations at low $|\Delta y|$ for the lightflavor sample, a possible indication of strangeness ordering along the quark-antiquark axis for associated $K^{+} K^{-}$pairs, similar to the baryon number ordering observed for $\mathrm{p} \overline{\mathrm{p}}$ pairs. Such an effect is expected to be diluted at high momentum by an effect of the opposite sign due to triplets of high-momentum kaons produced in $s \bar{s}$ events. We do not observe such a signal in the heavy flavor events, possibly due to limited statistics, dilution due to heavy hadron decays that include a $K^{+}$and a $K^{-}$, and/or dilution due to the negative difference from opposite hemisphere pairs, that populate a lower $|\Delta y|$ region in heavy-flavor events than in light-flavor events.

In fig. 11 we show the difference for $\pi^{+} \pi^{-}$pairs in two bins of $p_{\max }$ for the lightflavor and $c \bar{c}$ samples separately. For large $p_{\max }$ we observe a negative difference at high $|\Delta y|$ in the light-flavor sample, as expected from leading pion production. In the $c \bar{c}$ events, there are positive differences in both bins of $p_{\max }$ at high $\left|\Delta y^{+-}\right|$and also in the low $p_{\max }$ bin at low $\left|\Delta y^{+-}\right|$, all of which can be attributed to pairs involving a $\pi^{+}$from the $D$ meson decay and a $\pi^{-}$from the $\bar{D}$ meson decay. The predictions of the 


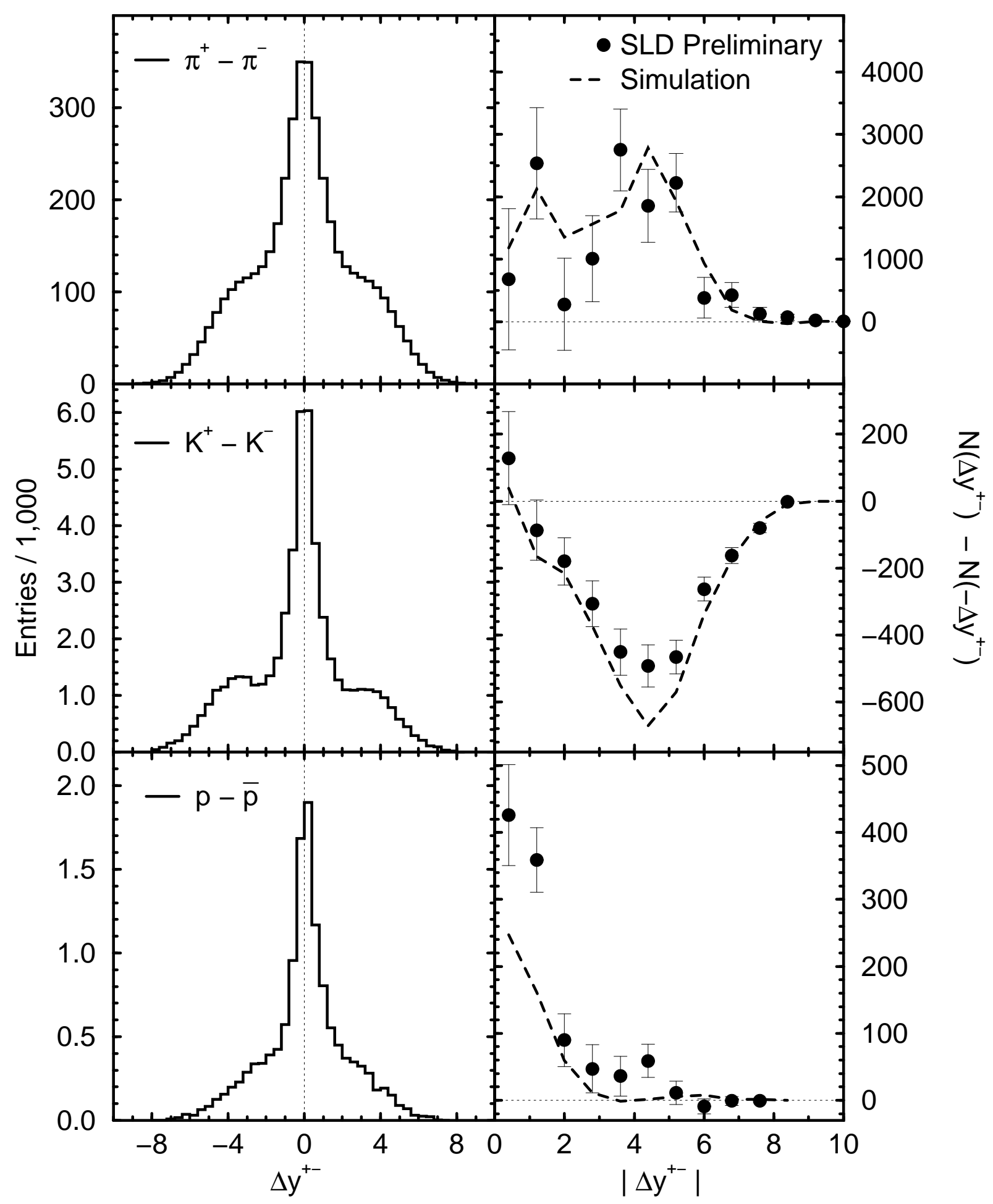

Figure 8: Distributions (left) of the difference between the signed rapidities of positively and negatively charged identified hadrons of the same type. The differences (right) between the right and left sides of the distributions, compared with the prediction of the Monte Carlo simulation. 


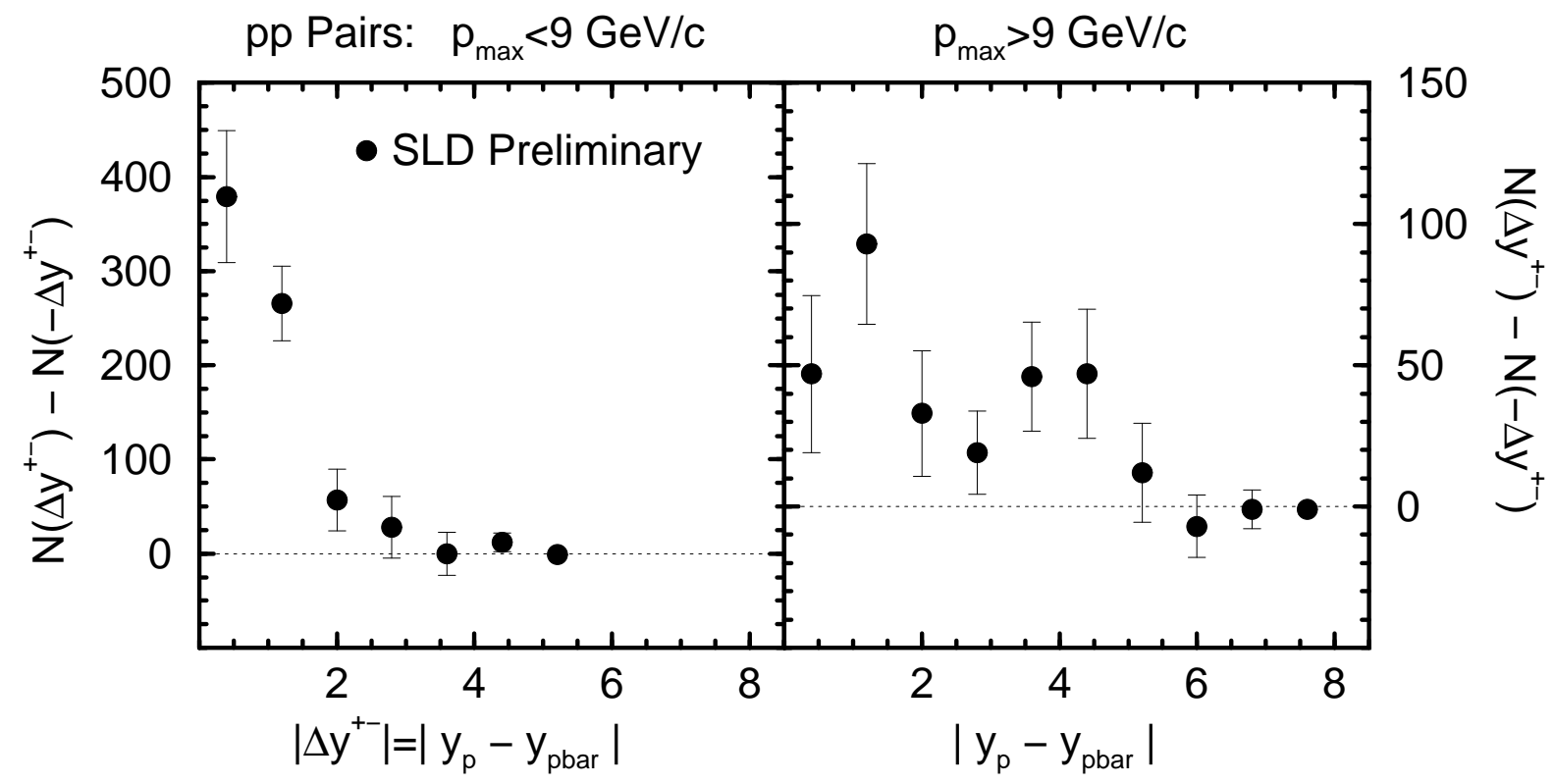

Figure 9: Differences between the positive and negative sides of the distributions of $y_{\mathrm{p}}-y_{\overline{\mathrm{p}}}$ at low (left) and high (right) momentum of the higher-momentum track.

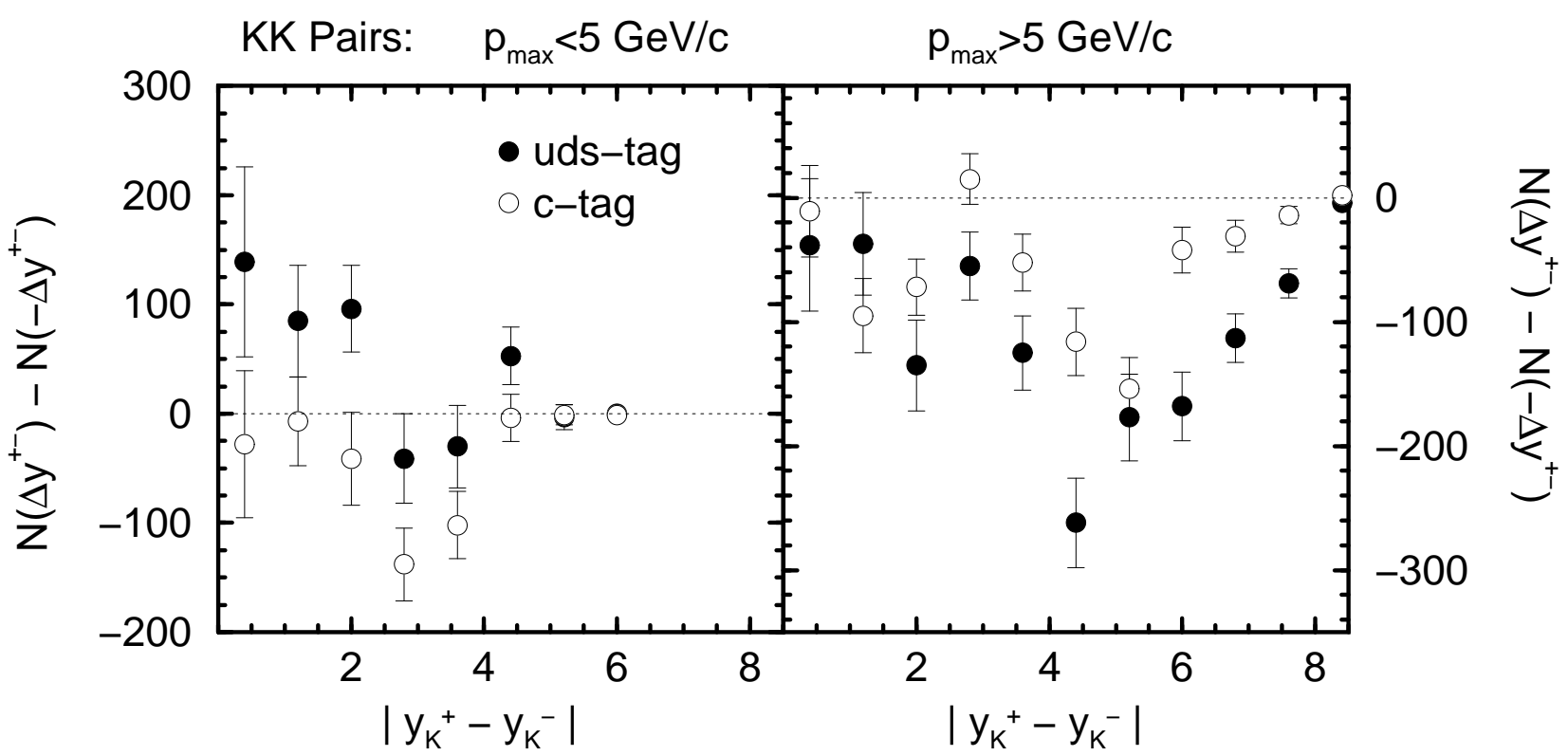

Figure 10: Differences between the positive and negative sides of the distribution of $y_{K^{+}}-y_{K^{-}}$for pairs produced at low (left) and high (right) momentum, in the light(dots) and $c$-tagged (open circles) samples. 


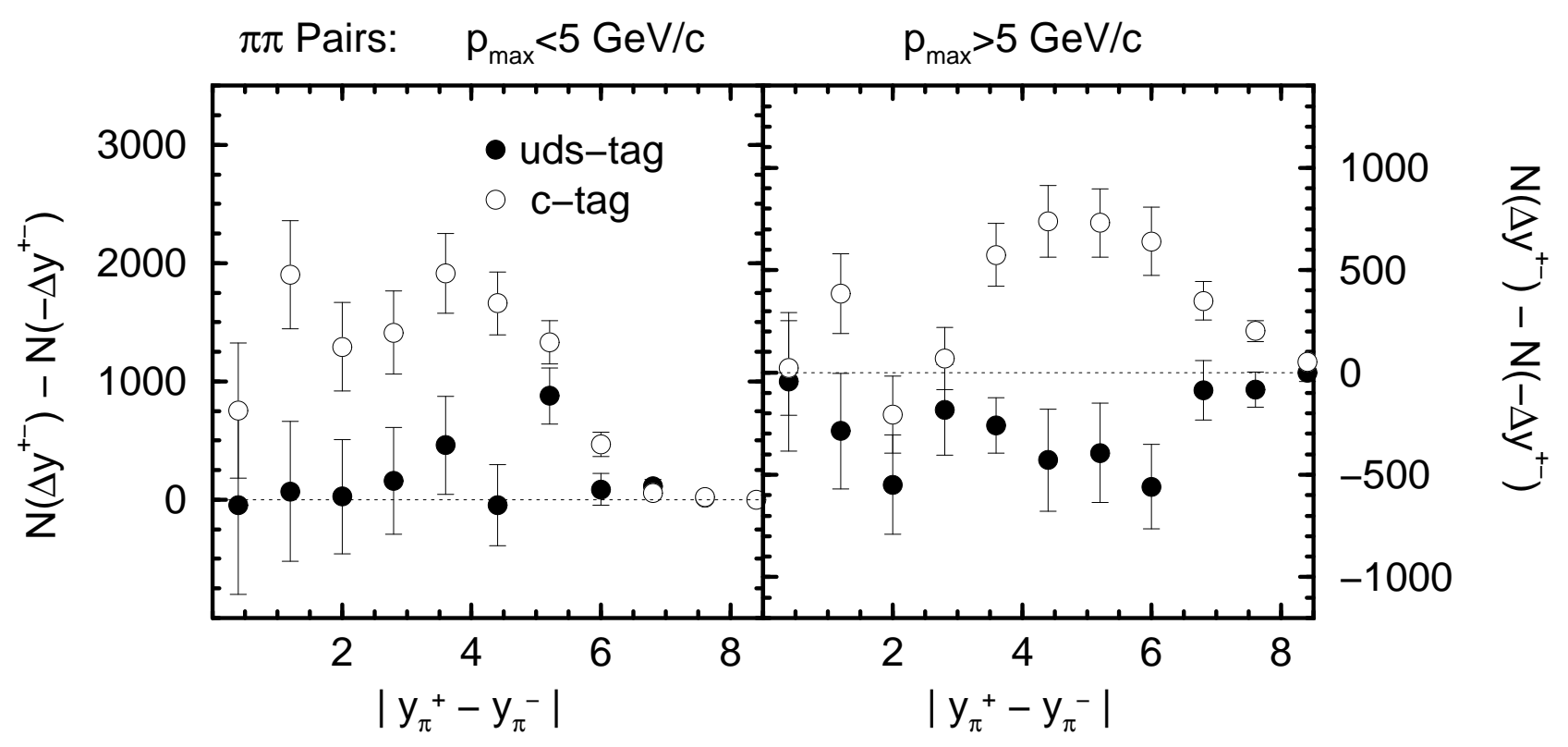

Figure 11: Differences between the positive and negative sides of the distribution of $y_{\pi^{+}}-y_{\pi^{-}}$at low (left) and high (right) momentum, in the light- (dots) and $c$-tagged (open circles) samples.

simulation are consistent with these data.

For unlike particles, both opposite-charge and same-charge pairs may be of interest. We define the ordered difference as the rapidity of the heavier particle minus that of the lighter particle multiplied by the charge of the heavier particle. That is, $\Delta y^{+-}=$ $y_{K^{+}}-y_{\pi^{-}}$or $y_{\pi^{-}}-y_{K^{+}}, \Delta y^{++}=y_{K^{+}}-y_{\pi^{+}}, \Delta y^{--}=y_{\pi^{-}}-y_{K^{-}}$, etc. In fig. 12 we show the distributions of $\Delta y^{+-}$for opposite-charge pairs of each of the three combinations of unlike particles, as well as the sum of the $\Delta y^{++}$and $\Delta y^{--}$distributions for the corresponding same-charge pairs. A significant negative asymmetry is observed for $\pi K$ pairs of both opposite- and same-charge at all $\Delta y$, which may simply be due the combination of leading kaons and randomly selected pions. A similar effect in $K \mathrm{p}$ pairs can be attributed to leading kaons combined with randomly chosen protons. In this case the asymmetry is negative for opposite-charge pairs and positive for samecharge pairs, as expected given our sign convention. In the case of $\pi p$ pairs there is a small positive asymmetry for both opposite- and same-charge pairs. The asymmetries predicted by the simulation are also shown; they are consistent with the $\mathrm{p} K^{+}$data, but overestimate the magnitudes of the effects for $\mathrm{p} K^{-}, K^{+} \pi^{-}$and $K^{+} \pi^{+}$pairs slightly. The latter two differences are expected in light of the absence of a $\pi K$ correlation in the simulation of light-flavor events noted above. A small negative asymmetry is predicted 
for $\pi$ p pairs, which is inconsistent with the observed positive asymmetry.

\section{Conclusion}

We have presented a preliminary study of rapidity differences between pairs of identified charged pions, kaons and protons in light-flavor hadronic $Z^{0}$ decays. The SLD Cherenkov Ring Imaging Detector was used to select clean samples of identified charged hadrons, and the Vertex Detector was used to investigate the flavor dependence of the results.

We observe excesses of opposite-charge over same-charge pairs for all pair combinations at low values of the absolute rapidity difference, indicating that there is a high degree of local conservation of baryon number, strangeness and electric charge in the fragmentation process. The predictions of the JETSET fragmentation model are found to provide a qualitative description of the data, although they fail to describe the forms of some of the correlations in detail. The range of these short-range correlations was studied as a function of momentum; the variations found were reproduced by the simulation, verifying within the context of the JETSET model that the correlations are scale invariant.

We observe a large excess of high momentum $K^{+} K^{-}$pairs over same-charge kaon pairs at large values of the absolute rapidity difference, and the effect is larger for higher momenta, as expected from leading kaon production in $s \bar{s}$ events. Weaker correlations are observed for protons, indicating that events with a leading baryon in one jet, a leading antibaryon in the other and no additional baryons do not contribute significantly to baryon production in $e^{+} e^{-}$annihilations. Significant long-range correlations are observed between opposite-charge pairs of all combinations in light-flavor events; in $c \bar{c}$ events, long-range $\pi \pi, \pi \mathrm{p}$ and $K K$ correlations are observed, along with a strong $\pi K$ anticorrelation. The simulation provides a good description of the data in general, but does not predict the long-range $\pi K$ correlation in light-flavor events.

We have studied distributions of rapidity signed so that positive rapidity corresponds to the quark (rather than antiquark) direction. Differences between signed rapidity distributions for positive and negative hadrons of all three species are observed,

giving further evidence for leading production of charged pions kaons and protons. The 


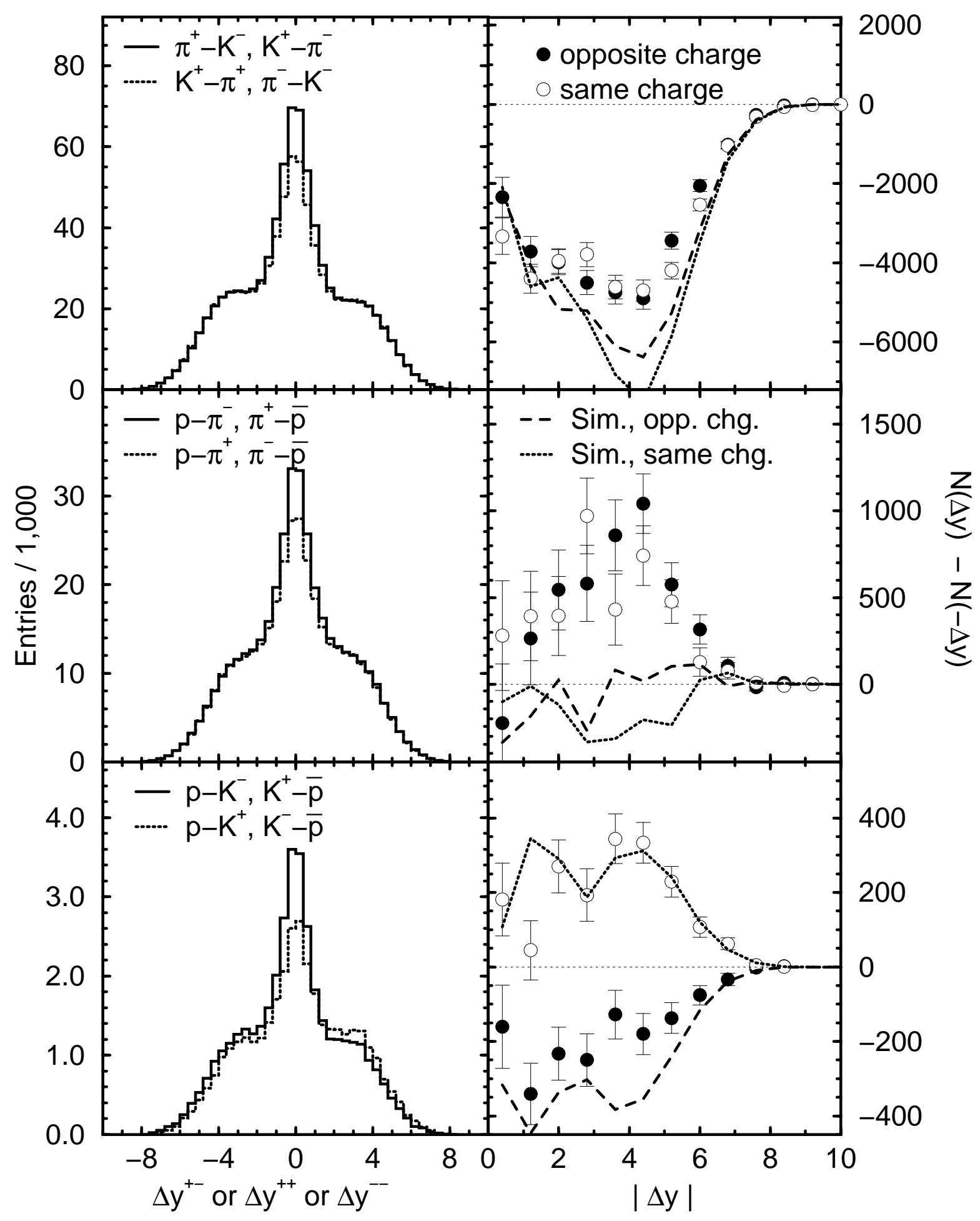

Figure 12: Distributions (left) of the difference between the signed rapidities of positively and negatively charged identified hadrons of different types. The differences (right) between the right and left sides of the distributions, compared with the predictions of the Monte Carlo simulation. 
distribution of the difference between the signed rapidities of $K^{+}$and $K^{-}$shows a large asymmetry at large values of the absolute rapidity difference, a direct indication that the long-range correlated $K K$ pairs are dominated by $s \bar{s}$ events. A similar but smaller difference for $\pi^{+} \pi^{-}$pairs indicates roughly equal production of leading pions in $u \bar{u}$ and $d \bar{d}$ events. There is a large asymmetry at small rapidity difference for $\mathrm{p} \overline{\mathrm{p}}$ pairs, a clear indication of ordering of baryons along the event axis. A similar effect is observed for $K^{+} K^{-}$pairs at low momentum in light-flavor events.

\section{Acknowledgements}

We thank the personnel of the SLAC accelerator department and the technical staffs of our collaborating institutions for their outstanding efforts on our behalf.

*Work supported by Department of Energy contracts: DE-FG02-91ER40676 (BU), DE-FG03-91ER40618 (UCSB), DE-FG03-92ER40689 (UCSC), DE-FG03-93ER40788 (CSU), DE-FG02-91ER40672 (Colorado), DE-FG02-91ER40677 (Illinois), DE-AC03-76SF00098 (LBL), DE-FG02-92ER40715 (Massachusetts), DE-FC02-94ER40818 (MIT), DE-FG03-96ER40969 (Oregon), DE-AC03-76SF00515 (SLAC), DE-FG05-91ER40627 (Tennessee), DE-FG02-95ER40896 (Wisconsin), DE-FG02-92ER40704 (Yale); National Science Foundation grants: PHY-91-13428 (UCSC), PHY-89-21320 (Columbia), PHY-9204239 (Cincinnati), PHY-95-10439 (Rutgers), PHY-88-19316 (Vanderbilt), PHY-92-03212 (Washington); The UK Particle Physics and Astronomy Research Council (Brunel, Oxford and RAL); The Istituto Nazionale di Fisica Nucleare of Italy (Bologna, Ferrara, Frascati, Pisa, Padova, Perugia); The Japan-US Cooperative Research Project on High Energy Physics (Nagoya, Tohoku); The Korea Research Foundation (Soongsil, 1997).

\section{References}

[1] TPC Collab., H. Aihara et al., Phys. Rev. Lett. 57 (1986) 3140;

TASSO Collab., R. Brandelik et al., Phys. Lett. B139 (1984) 126;

ALEPH Collab., D. Buskulic et al., Z. Phys. C64 (1994) 361;

DELPHI Collab., P. Abreu et al., Phys. Lett. B416 (1998) 247;

OPAL Collab., P.D. Acton et al., Phys. Lett. B305 (1993) 415. 
[2] TPC Collab., H. Aihara et al., Phys. Rev. Lett. 53 (1984) 2199;

TASSO Collab., R. Brandelik et al., Phys. Lett. B100 (1981) 357;

A. Breakstone et al., Z. Phys. C25 (1984) 21.

[3] SLD Design Report, SLAC-Report 273 (1984).

[4] SLD Collaboration: K. Abe et al., Phys. Rev. D51 (1995) 962.

[5] M. D. Hildreth et al., Nucl. Instr. Meth. A367 (1995) 111.

[6] C.J.S. Damerell et. al., Nucl. Instr. Meth. A288 (1990) 236.

C.J.S. Damerell et. al., Nucl. Instr. Meth. A400 (1997) 287.

[7] K. Abe et al., Nucl. Inst. Meth. A343 (1994) 74.

[8] SLD Collab., K. Abe et al., Phys. Rev. D59 52001.

[9] S. Brandt et al., Phys. Lett. 12 (1964) 57.

E. Farhi, Phys. Rev. Lett. 39 (1977) 1587.

[10] D. Axen et al., Nucl. Inst. Meth. A328 (1993) 472.

[11] SLD Collab., K. Abe et al., Phys. Rev. D53 (1996) 1023.

[12] T. Sjöstrand, Comput. Phys. Commun. 82 (1994) 74.

[13] P. N. Burrows, Z. Phys. C41 (1988) 375.

OPAL Collaboration, M.Z. Akrawy et al., Z. Phys. C47 (1990) 505.

[14] SLD Collaboration, K. Abe et al., Phys. Rev. Lett. 79 (1997) 590.

[15] R. Brun et al., Report No. CERN-DD/EE/84-1 (1989).

[16] SLD Collaboration, K. Abe et al., Phys. Rev. Lett. 74 (1995) 2895.

[17] K. Abe et al., Nucl. Inst. and Meth. A371 (1996) 195.

[18] SLD Collab., K. Abe et al., Phys. Rev. Lett. 78 (1997) 3442. 


\section{${ }^{* *}$ List of Authors}

Kenji Abe, ${ }^{(21)}$ Koya Abe, ${ }^{(33)}$ T. Abe, ${ }^{(29)}$ I.Adam, ${ }^{(29)}$ T. Akagi, ${ }^{(29)}$ N. J. Allen, ${ }^{(5)}$ W.W. Ash, ${ }^{(29)}$ D. Aston, ${ }^{(29)}$ K.G. Baird, ${ }^{(17)}$ C. Baltay, ${ }^{(40)}$ H.R. Band, ${ }^{(39)}$ M.B. Barakat, ${ }^{(16)}$ O. Bardon, ${ }^{(19)}$ T.L. Barklow, ${ }^{(29)}$ G. L. Bashindzhagyan, ${ }^{(20)}$ J.M. Bauer, ${ }^{(18)}$ G. Bellodi, ${ }^{(23)}$ R. Ben-David, ${ }^{(40)}$ A.C. Benvenuti, ${ }^{(3)}$ G.M. Bilei, ${ }^{(25)}$ D. Bisello, ${ }^{(24)}$ G. Blaylock, ${ }^{(17)}$ J.R. Bogart, ${ }^{(29)}$ G.R. Bower, ${ }^{(29)}$ J. E. Brau, ${ }^{(22)}$ M. Breidenbach, ${ }^{(29)}$ W.M. Bugg, ${ }^{(32)}$ D. Burke, ${ }^{(29)}$ T.H. Burnett, ${ }^{(38)}$ P.N. Burrows, ${ }^{(23)}$

A. Calcaterra, ${ }^{(12)}$ D. Calloway, ${ }^{(29)}$ B. Camanzi, ${ }^{(11)}$ M. Carpinelli, ${ }^{(26)}$ R. Cassell, ${ }^{(29)}$

R. Castaldi, ${ }^{(26)}$ A. Castro, ${ }^{(24)}$ M. Cavalli-Sforza, ${ }^{(35)}$ A. Chou, ${ }^{(29)}$ E. Church, ${ }^{(38)}$ H.O. Cohn, ${ }^{(32)}$ J.A. Coller, ${ }^{6}{ }^{6}$ M.R. Convery, ${ }^{(29)}$ V. Cook, ${ }^{(38)}$ R. Cotton, ${ }^{(5)}$ R.F. Cowan, ${ }^{(19)}$ D.G. Coyne, ${ }^{(35)}$ G. Crawford, ${ }^{(29)}$ C.J.S. Damerell, ${ }^{(27)}$

M. N. Danielson, ${ }^{(8)}$ M. Daoudi, ${ }^{(29)}$ N. de Groot, ${ }^{(4)}$ R. Dell'Orso, ${ }^{(25)}$ P.J. Dervan, ${ }^{(5)}$ R. de Sangro, ${ }^{(12)}$ M. Dima, ${ }^{(10)}$ A. D'Oliveira, ${ }^{(7)}$ D.N. Dong, ${ }^{(19)}$ M. Doser, ${ }^{(29)}$ R. Dubois, ${ }^{(29)}$ B.I. Eisenstein, ${ }^{(13)}$ V. Eschenburg, ${ }^{(18)}$ E. Etzion, ${ }^{(39)}$ S. Fahey, ${ }^{(8)}$ D. Falciai, ${ }^{(12)}$ C. Fan, ${ }^{(8)}$ J.P. Fernandez, ${ }^{(35)}$ M.J. Fero, ${ }^{(19)}$ K.Flood, ${ }^{(17)}$ R. Frey, ${ }^{(22)}$

J. Gifford, ${ }^{(36)}$ T. Gillman, ${ }^{(27)}$ G. Gladding, ${ }^{(13)}$ S. Gonzalez, ${ }^{(19)}$ E. R. Goodman, ${ }^{(8)}$ E.L. Hart, ${ }^{(32)}$ J.L. Harton, ${ }^{(10)}$ A. Hasan, ${ }^{(5)}$ K. Hasuko, ${ }^{(33)}$ S. J. Hedges, ${ }^{(6)}$

S.S. Hertzbach, ${ }^{(17)}$ M.D. Hildreth, ${ }^{(29)}$ J. Huber, ${ }^{(22)}$ M.E. Huffer, ${ }^{(29)}$ E.W. Hughes, ${ }^{(29)}$ X.Huynh, ${ }^{(29)}$ H. Hwang, ${ }^{(22)}$ M. Iwasaki, ${ }^{(22)}$ D. J. Jackson, ${ }^{(27)}$ P. Jacques, ${ }^{(28)}$ J.A. Jaros, ${ }^{(29)}$ Z.Y. Jiang, ${ }^{(29)}$ A.S. Johnson, ${ }^{(29)}$ J.R. Johnson, ${ }^{(39)}$ R.A. Johnson, ${ }^{(7)}$

T. Junk, ${ }^{(29)}$ R. Kajikawa, ${ }^{(21)}$ M. Kalelkar, ${ }^{(28)}$ Y. Kamyshkov, ${ }^{(32)}$ H.J. Kang, ${ }^{(28)}$ I. Karliner, ${ }^{(13)}$ H. Kawahara, ${ }^{(29)}$ Y. D. Kim, ${ }^{(30)}$ M.E. King, ${ }^{(29)}$ R. King, ${ }^{(29)}$ R.R. Kofler, ${ }^{(17)}$ N.M. Krishna, ${ }^{(8)}$ R.S. Kroeger, ${ }^{(18)}$ M. Langston, ${ }^{(22)}$ A. Lath, ${ }^{(19)}$ D.W.G. Leith, ${ }^{(29)}$ V. Lia, ${ }^{(19)}$ C.Lin, ${ }^{(17)}$ M.X. Liu, ${ }^{(40)}$ X. Liu, ${ }^{(35)}$ M. Loreti, ${ }^{(24)}$ A. Lu, ${ }^{(34)}$ H.L. Lynch, ${ }^{(29)}$ J. Ma, ${ }^{(38)}$ G. Mancinelli, ${ }^{(28)}$ S. Manly, ${ }^{(40)}$ G. Mantovani, ${ }^{(25)}$ T.W. Markiewicz, ${ }^{(29)}$ T. Maruyama, ${ }^{(29)}$ H. Masuda, ${ }^{(29)}$ E. Mazzucato, ${ }^{(11)}$ A.K. McKemey, ${ }^{(5)}$ B.T. Meadows, ${ }^{(7)}$ G. Menegatti, ${ }^{(11)}$ R. Messner, ${ }^{(29)}$ P.M. Mockett, ${ }^{(38)}$ K.C. Moffeit, ${ }^{(29)}$ T.B. Moore, ${ }^{(40)}$ M.Morii, ${ }^{(29)}$ D. Muller, ${ }^{(29)}$ V.Murzin, ${ }^{(20)}$ T. Nagamine, ${ }^{(33)}$ S. Narita, ${ }^{(33)}$ U. Nauenberg, ${ }^{(8)}$ H. Neal, ${ }^{(29)}$ M. Nussbaum, ${ }^{(7)}$ N.Oishi, ${ }^{(21)}$ D. Onoprienko, ${ }^{(32)}$ L.S. Osborne, ${ }^{(19)}$ R.S. Panvini, ${ }^{(37)}$ C. H. Park, ${ }^{(31)}$ T.J. Pavel, ${ }^{(29)}$ I. Peruzzi, ${ }^{(12)}$ M. Piccolo, ${ }^{(12)}$ L. Piemontese, ${ }^{(11)}$

K.T. Pitts, ${ }^{(22)}$ R.J. Plano, ${ }^{(28)}$ R. Prepost, ${ }^{(39)}$ C.Y. Prescott, ${ }^{(29)}$ G.D. Punkar, ${ }^{(29)}$ J. Quigley, ${ }^{(19)}$ B.N. Ratcliff, ${ }^{(29)}$ T.W. Reeves, ${ }^{(37)}$ J. Reidy, ${ }^{(18)}$ P.L. Reinertsen, ${ }^{(35)}$ P.E. Rensing, ${ }^{(29)}$ L.S. Rochester, ${ }^{(29)}$ P.C. Rowson, ${ }^{(9)}$ J.J. Russell, ${ }^{(29)}$ O.H. Saxton, ${ }^{(29)}$

T. Schalk, ${ }^{(35)}$ R.H. Schindler, ${ }^{(29)}$ B.A. Schumm, ${ }^{(35)}$ J. Schwiening, ${ }^{(29)}$ S. Sen, ${ }^{(40)}$

V.V. Serbo, ${ }^{(29)}$ M.H. Shaevitz, ${ }^{(9)}$ J.T. Shank, ${ }^{(6)}$ G. Shapiro, ${ }^{(15)}$ D.J. Sherden, ${ }^{(29)}$ K. D. Shmakov, ${ }^{(32)}$ C. Simopoulos, ${ }^{(29)}$ N.B. Sinev, ${ }^{(22)}$ S.R. Smith, ${ }^{(29)}$ M. B. Smy, ${ }^{(10)}$ J.A. Snyder, ${ }^{(40)}$ H. Staengle, ${ }^{(10)}$ A. Stahl, ${ }^{(29)}$ P. Stamer, ${ }^{(28)}$ H. Steiner, ${ }^{(15)}$ R. Steiner, ${ }^{(1)}$ M.G. Strauss, ${ }^{(17)}$ D. Su, ${ }^{(29)}$ F. Suekane, ${ }^{(33)}$ A. Sugiyama, ${ }^{(21)}$ S. Suzuki, ${ }^{(21)}$ M. Swartz, ${ }^{(14)}$ A. Szumilo, ${ }^{(38)}$ T. Takahashi, ${ }^{(29)}$ F.E. Taylor, ${ }^{(19)}$ J. Thom, ${ }^{(29)}$ E. Torrence, ${ }^{(19)}$ N. K. Toumbas, ${ }^{(29)}$ T. Usher, ${ }^{(29)}$ C. Vannini, ${ }^{(26)}$ J. Va'vra, ${ }^{(29)}$ E. Vella, ${ }^{(29)}$ J.P. Venuti, ${ }^{(37)}$ R. Verdier, ${ }^{(19)}$ P.G. Verdini, ${ }^{(26)}$ 
D. L. Wagner, ${ }^{(8)}$ S.R. Wagner, ${ }^{(29)}$ A.P. Waite, ${ }^{(29)}$ S. Walston, ${ }^{(22)}$ J.Wang, ${ }^{(29)}$ S.J. Watts, ${ }^{(5)}$ A.W. Weidemann, ${ }^{(32)}$ E. R. Weiss ${ }^{(38)}$ J.S. Whitaker ${ }^{(6)}$ S.L. White, ${ }^{(32)}$ F.J. Wickens, ${ }^{(27)}$ B. Williams, ${ }^{\left({ }^{(}\right)}$D.C. Williams, ${ }^{(19)}$ S.H. Williams, ${ }^{(29)}$ S. Willocq, ${ }^{(17)}$ R.J. Wilson, ${ }^{(10)}$ W.J. Wisniewski, ${ }^{(29)}$ J. L. Wittlin, ${ }^{(17)}$ M. Woods, ${ }^{(29)}$ G.B. Word, ${ }^{(37)}$ T.R. Wright, ${ }^{(39)}$ J. Wyss, ${ }^{(24)}$ R.K. Yamamoto, ${ }^{(19)}$ J.M. Yamartino, ${ }^{(19)}$ X. Yang, ${ }^{(22)}$ J. Yashima, ${ }^{(33)}$ S.J. Yellin, ${ }^{(34)}$ C.C. Young, ${ }^{(29)}$ H. Yuta, ${ }^{(2)}$ G. Zapalac,${ }^{(39)}$ R.W. Zdarko, ${ }^{(29)}$ J. Zhou. ${ }^{(22)}$

(1) Adelphi University, Garden City, New York 11530,

${ }^{(2)}$ Aomori University, Aomori, 030 Japan,

(3) INFN Sezione di Bologna, I-40126, Bologna Italy,

(4) University of Bristol, Bristol, U.K.,

${ }^{(5)}$ Brunel University, Uxbridge, Middlesex, UB8 3PH United Kingdom,

${ }^{(6)}$ Boston University, Boston, Massachusetts 02215,

(7) University of Cincinnati, Cincinnati, Ohio 45221,

(8) University of Colorado, Boulder, Colorado 80309,

${ }^{(9)}$ Columbia University, New York, New York 10533,

${ }^{(10)}$ Colorado State University, Ft. Collins, Colorado 80523,

${ }^{(11)}$ INFN Sezione di Ferrara and Universita di Ferrara, I-44100 Ferrara, Italy,

${ }^{(12)}$ INFN Lab. Nazionali di Frascati, I-00044 Frascati, Italy,

(13) University of Illinois, Urbana, Illinois 61801,

(14) Johns Hopkins University, Baltimore, MD 21218-2686,

${ }^{(15)}$ Lawrence Berkeley Laboratory, University of California, Berkeley, California 94720 ,

(16) Louisiana Technical University - Ruston,LA 71272,

(17) University of Massachusetts, Amherst, Massachusetts 01003,

(18) University of Mississippi, University, Mississippi 38677,

(19) Massachusetts Institute of Technology, Cambridge, Massachusetts 02139,

${ }^{(20)}$ Institute of Nuclear Physics, Moscow State University, 119899, Moscow Russia,

(21) Nagoya University, Chikusa-ku, Nagoya 464 Japan,

${ }^{(22)}$ University of Oregon, Eugene, Oregon 97403,

(23) Oxford University, Oxford, OX1 3RH, United Kingdom,

(24) INFN Sezione di Padova and Universita di Padova I-35100, Padova, Italy,

(25) INFN Sezione di Perugia and Universita di Perugia, I-06100 Perugia, Italy,

(26) INFN Sezione di Pisa and Universita di Pisa, I-56010 Pisa, Italy,

${ }^{(27)}$ Rutherford Appleton Laboratory, Chilton, Didcot, Oxon OX11 OQX United Kingdom, 
(28) Rutgers University, Piscataway, New Jersey 08855,

(29) Stanford Linear Accelerator Center, Stanford University, Stanford, California 94309,

(30) Sogang University, Seoul, Korea,

(31) Soongsil University, Seoul, Korea 156-743,

(32) University of Tennessee, Knoxville, Tennessee 37996,

(33) Tohoku University, Sendai 980, Japan,

(34) University of California at Santa Barbara, Santa Barbara, California 93106, (35) University of California at Santa Cruz, Santa Cruz, California 95064,

(36) University of Victoria, Victoria, B.C., Canada, V8W 3P6,

(37) Vanderbilt University, Nashville, Tennessee 37235,

(38) University of Washington, Seattle, Washington 98105,

(39) University of Wisconsin, Madison, Wisconsin 53706,

(40) Yale University, New Haven, Connecticut 06511. 